\title{
Seasonality and Stochastic Trends in German Consumption and Income, 1960.1-1987.4
}

\author{
Philip Hans Franses and Richard PaAp \\ Econometric Institute and Tinbergen Institute, Erasmus University Rotterdam, P.O. Box 1738, \\ NL-3000 DR Rotterdam, The Netherlands
}

\begin{abstract}
The quarterly time series of German consumption and income are analyzed with respect to seasonality and stochastic trends. It emerges that both variables can be appropriately described by a periodically integrated autoregression. An implication is that the stochastic trend and the seasonal fuctuations are not independent for each of the univariate series. In order to test for cointegration across the two series, we propose several methods which take account of the relationship between seasons and trends in the univariate series. Some of these methods boil down to extracting the stochastic trend from the univariate series in a first step and to relating these trends using cointegration techniques in a second step. Another method is an extension of the Johansen cointegration testing approach to periodic vector autoregressions. Monte Carlo simulations are used to evaluate the empirical performance of the various methods. The main empirical result is that only in the first quarter there seems to be cointegration between German consumption and income.
\end{abstract}

JEL Classification System-Numbers: C22, C32

\section{Introduction and Summary}

In this paper we study the seasonal and stochastic trend properties of German consumption and income over the period 1960.1-1987.4. This analysis will focus on the univariate as well as the multivariate properties of the two series. For the univariate analysis we use the model selection strategy proposed and evaluated in Franses and Paap (1993). This strategy amounts to estimating periodic autoregressive [PAR] time series models for each of the series, and, in case periodicity cannot be rejected, to testing for the presence of stochastic trends within the PAR framework. For the German data we find that a PAR model of order one adequately describes both series, and that each series has a stochastic trend. However, we show that this stochastic trend cannot be removed by taking first order differences because we find that German consumption and income are so-called periodically integrated, and hence that a seasonally varying differencing filter is needed.

A main feature of periodic integration is that the stochastic trend and the seasonal fluctuations are not independent. Loosely speaking, a change in the direction of the stochastic trend causes a change in the seasonal pattern. This dependency of seasonality on the stochastic trend establishes that common cointegration testing methods, which are typically applied to nonperiodic time 
series, may yield incorrect inference with respect to the presence of a cointegration relationship between consumption and income. In this paper we therefore propose and evaluate several methods to check for cointegration between periodically integrated time series. Some of these methods consist of two steps. The first step amounts to extracting the stochastic trends from each of the univariate series using a variety of approaches. In the second step we check for the presence of cointegration among these extracted stochastic trend series using wellknown cointegration testing methods. An alternative method which we propose in this paper is an extension of the Johansen cointegration approach to periodic vector autoregressions. Monte Carlo simulations are used to evaluate the empirical performance of all methods in small samples. The main empirical result we obtain is that there seems to be evidence in favor of cointegration between consumption and income in the first quarter only.

The outline of this paper is as follows. First, in section 2, we analyse the two univariate time series using periodic autoregressive models. In section 3 , we propose, evaluate and apply several methods to test for cointegration between German consumption and income. Some concluding remarks are given in the final section.

\section{Univariate Analysis}

The data we analyse in this paper are given in Lütkepohl (1991, table E.4.), where they are used as an illustration to periodic time series models. The consumption $c_{t}$ and $y_{t}$ series are the logs of the seasonally unadjusted (West-) German Real per Capita Personal Consumption Expenditures and Personal Disposable Income, 1960.1-1987.4. Graphs of the variables are displayed in figure 1.

It can be seen that both series show seasonal fluctuations around a trending pattern. It can also be observed that the seasonal patterns do not seem to be constant over time, although the changes seem to occur only slowly.

In the figures 2 and 3, we display the graphs of the first order differenced time series $\Delta_{1} c_{t}$ and $\Delta_{1} y_{t}$, where $\Delta_{i}$ is defined by $\Delta_{i} z_{t}=\left(1-B^{i}\right) z_{t}$, where $B$ is the familiar backward shift operator defined by $B^{i} z_{t}=z_{t-i}$. Note that these graphs do not connect the quarterly observations, but that they connect the data points corresponding to each of the seasons $1,2,3$ and 4 . From figure 2 one can observe that the mean of the $\Delta_{1}$ transformed consumption series is not constant. In fact, the mean of $\Delta_{1} c_{t}$ in the fourth quarter seems to be lower after around 1974, and the mean in the second quarter seems lower after 1980. Hence, the changes in the seasonal fluctuations in consumption may coincide with two major business cycle fluctuations, which are likely to be established by the two oil shocks. Figure 3 indicates that the $\Delta_{1} y_{t}$ series contains trend-like be- 


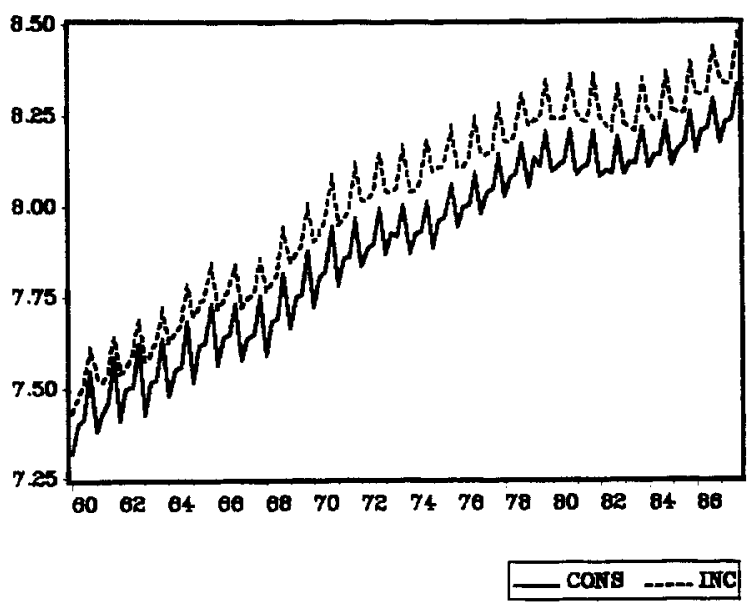

Fig. 1. German consumption and income

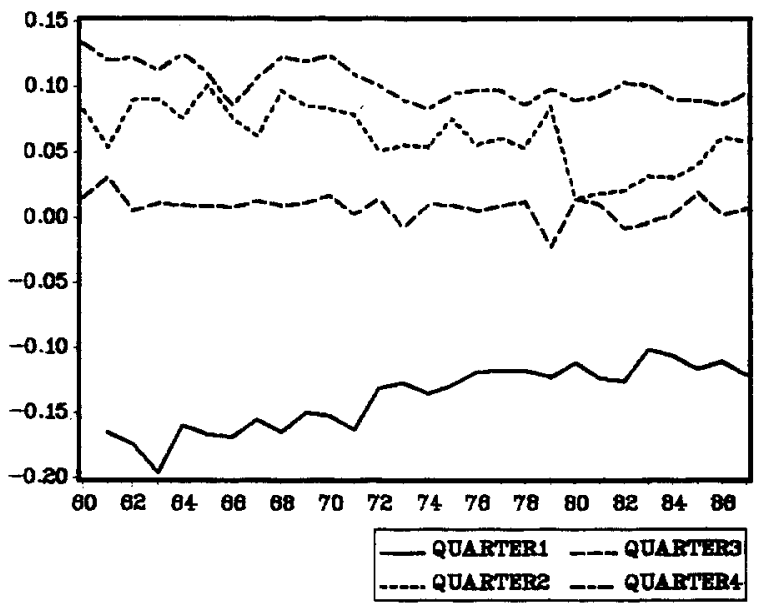

Fig. 2. First differences of log consumption

havior that varies with the seasons. In summary, the visual evidence obtained from these figures is that the $\Delta_{1}$ filter may not be suitable to remove stochastic trend fluctuations from each of the univariate time series.

To formally investigate the seasonality and stochastic trend properties of the two univariate time series, it has appeared useful to consider the class of periodic autoregressive [PAR] time series models, see Franses and Paap (1993). PAR models allow the autoregressive parameters to vary with the season, and hence nest nonperiodic AR models. The general PAR $(p)$ model for a quarterly observed time series $z_{t}, t=1, \ldots, n$, can be written as 


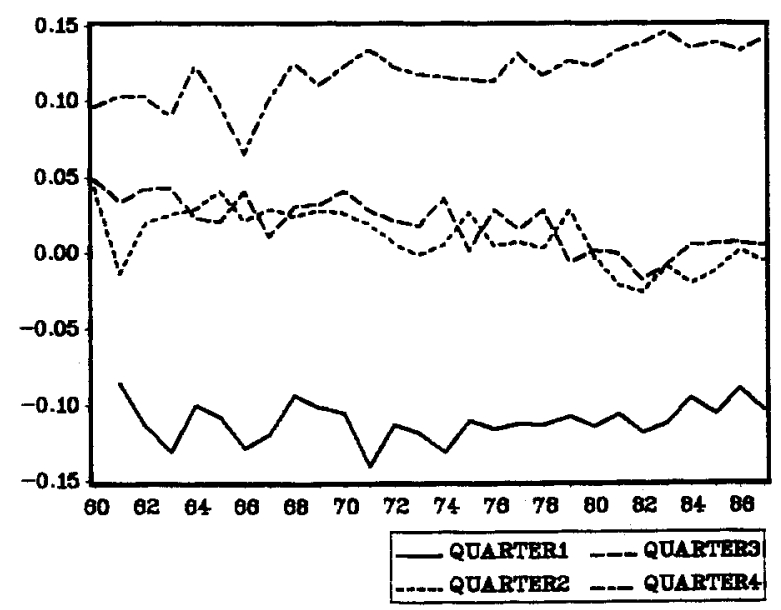

Fig. 3. First differences of log income

$$
z_{t}=\sum_{s=1}^{4} \mu_{s} D_{s t}+\sum_{s=1}^{4} \psi_{1 s} D_{s t} z_{t-1}+\cdots+\sum_{s=1}^{4} \psi_{p s} D_{s t} z_{t-p}+\varepsilon_{t}
$$

where $D_{s t}$ are the conventional seasonal dummy variables, $\varepsilon_{t}$ is an error term usually assumed to be a standard white noise process, and where $\mu_{s}, \psi_{1 s}, \ldots$, $\psi_{p s}$ are parameters that can take values which differ across the seasons. When all $\psi_{j s}$ equal $\psi_{j}$, the model (1) reduces to a nonperiodic $\operatorname{AR}(p)$ process with seasonally varying intercept terms. The model in (1) can be modified by replacing $\varepsilon_{t}$ by $\varepsilon_{s t}$, i.e. a seasonal heteroskedastic error process. Note that (1) also allows varying autoregressive model orders, as well as varying subset models since not all $\psi_{j s}$ have to be unequal to zero. The parameters in (1) can be estimated via ordinary least squares. Some early references to periodic AR models are Jones and Brelsford (1967), Pagano (1978) and Tiao and Grupe (1980).

\section{A Model Selection Strategy}

The model selection strategy proposed in Franses and Paap (1993) consists of four steps. In the first step the lag order $p$ of a $\operatorname{PAR}(p)$ is determined. Note that this $p$ is the maximum value of the possibly varying orders $p_{s}$, where $p_{s}$ denotes the AR lag order in season $s ; s=1,2,3$, 4. In Franses and Paap (1993) it is shown through Monte Carlo simulations that using a sequence of $F$ type tests for the significance of the four $\psi_{k s}$, where $k=k^{*}, \ldots, p$ with $p \leq k^{*}$ and $k^{*}$ is set at some prespecified value like, e.g., 8 , yields the most favourable empirical performance. Given the empirical adequacy of a certain $\operatorname{PAR}(p)$ process, the second step is to test for periodicity in the $\operatorname{PAR}(p)$ process, i.e. to test the 
hypothesis that $\psi_{p s}=\psi_{p}$ for all $s$. In Boswijk and Franses (1994) it is shown that the Likelihood Ratio based $F$ test for this hypothesis has an asymptotic $F$ distribution under the null hypothesis. In case the null hypothesis cannot be rejected, one can proceed with an analysis of the nonperiodic $\operatorname{AR}(p)$ model using, e.g., the method to test for seasonal unit roots as advocated in Hylleberg et al. (1990).

In case the null hypothesis of nonperiodicity in the estimated $\operatorname{PAR}(p)$ process can be rejected, the third step is to check for the presence of unit roots. For this purpose it is convenient to rewrite (1) in the so-called vector of quarters [VQ] representation where we consider the $(4 \times 1)$ vector process $Z_{T}=$ $\left(Z_{1 T}, Z_{2 T}, Z_{3 T}, Z_{4 T}\right)^{\prime}$, with $Z_{s T}$ denoting the observation in season $s$ in year $T=1, \ldots, N, N=n / 4$, i.e.

$$
A_{0} Z_{T}=\mu+A_{1} Z_{T-1}+\cdots+A_{m} Z_{T-m}+\varepsilon_{T},
$$

where $A_{j}, j=0,1, \ldots, m$ are $(4 \times 4)$ parameter matrices and where $\mu$ and $\varepsilon_{T}$ are $(4 \times 1)$ parameter vectors corresponding to $\mu_{s}$ and $\varepsilon_{t}$ in (1). For the value of $m$ it applies that $m=1+[p / 4]$, where ['] means "integer value of". The $A_{j}$ matrices contain the parameters $\psi_{p s}$ in (1). Note that (2) is a multivariate time series model with constant parameters. The presence of unit roots in $z_{t}$ can be checked by solving the characteristic equation

$$
\left|A_{0}-A_{1} z-\cdots-A_{m} z^{m}\right|=0 \text {, }
$$

and by investigating whether one or more solutions to (3) are equal to unity.

The empirical analysis of a large number of macroeconomic time series in Franses and Paap (1993) indicates that in practice the value of $p$ in (1) is typically smaller than 4 , and hence that $m$ in (2) can be set equal to 1 . Furthermore, it is found that, typically, there seems at most one unit root in PAR models like (2). A formal test for the hypothesis of a single unit root versus no unit root can be performed using the test statistic

$$
\mathrm{BF}=\operatorname{sign}(g(\hat{\psi})-1)\left(n \cdot \log \left(R S S_{0} / R S S_{1}\right)\right)^{1 / 2},
$$

see Boswijk and Franses (1994). The $g(\psi)$ is a nonlinear function of the $\psi_{p s}$ parameters evaluated under the alternative hypothesis of no unit root, which follows from solving (3). A simple example is given below in (6). The $R S S_{0}$ and $R S S_{1}$ are the residual sums of squares under the null and alternative hypothesis. Given that the single unit root hypothesis involves a nonlinear parameter restriction on the $\psi_{j s}$ parameters, the $R S S_{0}$ is found after applying nonlinear least squares. In Boswijk and Franses (1994) it is shown that this BF test in (4) follows a standard Dickey-Fuller distribution under the null hypothesis of a single unit root. As an example, consider the PAR(1) process $z_{t}=\psi_{s} z_{t-1}+\varepsilon_{t}$, which can be written as $A_{0} Z_{T}=A_{1} Z_{T-1}+\varepsilon_{T}$ with 


$$
A_{0}=\left[\begin{array}{cccc}
1 & 0 & 0 & 0 \\
-\psi_{2} & 1 & 0 & 0 \\
0 & -\psi_{3} & 1 & 0 \\
0 & 0 & -\psi_{4} & 1
\end{array}\right] \text { and } A_{1}=\left[\begin{array}{cccc}
0 & 0 & 0 & \psi_{1} \\
0 & 0 & 0 & 0 \\
0 & 0 & 0 & 0 \\
0 & 0 & 0 & 0
\end{array}\right]
$$

The characteristic equation as in (3) for this PAR(1) process is

$$
\left|A_{0}-A_{1} z\right|=1-\psi_{1} \psi_{2} \psi_{3} \psi_{4} z=0 \text {. }
$$

Thus, $z_{t}$ has a single unit root when $g(\psi)=\psi_{1} \psi_{2} \psi_{3} \psi_{4}=1$. When $Z_{T}$ is found to have a single unit root, the $z_{t}$ process is called periodically integrated of order 1 [PI(1)]. In case of a PI(1) time series, the appropriate differencing filter to remove the stochastic trend is $\left(1-\psi_{s} B\right)$ instead of $(1-B)$. Note that under the restriction $\psi_{1} \psi_{2} \psi_{3} \psi_{4}=1$, some $\psi_{s}$ values can exceed 1 .

The fourth and final step in the model selection strategy proposed in Franses and Paap (1993) is to test the validity of specific parameter restrictions in the PI(1) model, i.e. in the model where the nonlinear restriction which corresponds to the unit root is imposed. An interesting restriction in (5) is for example that all $\psi_{s}$ are equal to unity. In that case the PAR process is said to have a nonseasonal unit root since the appropriate differencing filter becomes $(1-B)$. On the other hand, when all $\psi_{s}$ are equal to -1 , the periodic process has a seasonal unit root at the bi-annual frequency. It is shown in Boswijk and Franses (1994) that Likelihood Ratio based $F$ test statistics for such hypotheses have an asymptotic $F$ distribution under the null hypothesis.

\section{Some Empirical Results}

The application of the first step in the above model selection strategy for $c_{t}$ and $y_{t}$ yields that both univariate series can be described by a PAR(1) model. To save space we do not report on the detailed model selection results, which can be obtained from the authors upon request. Neither do we report on the results of diagnostic test results for the models in this paper. We only mention that all models estimated in this paper pass LM type diagnostic checks for the absence of residual autocorrelation at lags 1 and 1 through 4, of periodic autocorrelation in the residuals at lag 1, of ARCH effects of order 1 and 1 through 4, and for the absence of nonnormality in the estimated residuals. Finally, in all models we present in this paper we include a few dummy variables to exclude some severely outlying observations. For consumption these dummy variables concern 1961.2 and 1979.2 and 1979.3. It turns out that the latter two dummy variables can be combined to a $(\ldots, 0,1,-1,0, \ldots)$ dummy variable. For income we use a dummy variable for 1966.4. We checked whether the exclusion of these dummy variables has any impact on the relevant parameter estimates and we found to evidence of such an impact. Hence, the inclusion of the 
dummy variables should be seen as a way to ensure that the estimated residuals are approximately white noise.

For consumption we find the following parameter estimates after ordinary least squares,

$$
c_{t}=\hat{\mu}_{s}+\hat{\phi}_{s} c_{t-1}+\hat{\varepsilon}_{t},
$$

with

$$
\begin{array}{cccc}
\hat{\mu}_{1}=-0.899 & \hat{\mu}_{2}=0.625 & \hat{\mu}_{3}=0.098 & \hat{\mu}_{4}=0.435 \\
(0.070) & (0.064) & (0.065) & (0.066) \\
\hat{\phi}_{1}=1.095 & \hat{\phi}_{2}=0.928 & \hat{\phi}_{3}=0.989 & \hat{\phi}_{4}=0.958 \\
(0.009) & (0.008) & (0.008) & (0.008)
\end{array}
$$

where the figures in parentheses are standard errors. A regression of $\hat{\varepsilon}_{t}^{2}$ on a constant and three seasonal dummies yields a significant $F$ statistic value of 4.544. Some experimentation results in the observation that this high value is caused by only a few data points at the end of the sample. In fact, re-estimating (7) for the sample 60.1-85.4 yields an insignificant $F$ statistic. Hence, we conclude that we do not have to be modify (7) by including $\varepsilon_{s t}$ instead of $\varepsilon_{t}$.

The $F$ test statistic for the hypothesis $\phi_{s}=\phi$, for all $s$, obtains a value of 68.675. Note that under the null hypothesis $\phi_{s}=\phi$ it is assumed that all seasonality in $c_{t}$ is deterministic since the model becomes $(1-B) c_{t}=\mu_{s}+\varepsilon_{t}$. Even when we enlarge model (7) with $\kappa_{2} c_{t-2}, \ldots, \kappa_{8} c_{t-8}$, where the $\kappa_{j}$ parameters are not time-varying, the $F$ test statistic for the hypothesis $\phi_{s}=\phi$ obtains the highly significant value of 13.190 . Hence, we conjecture that an empirically adequate model for consumption is a PAR(1) process. The product $\hat{\phi}_{1} \hat{\phi}_{2} \hat{\phi}_{3} \hat{\phi}_{4}$ equals 0.962 . The value of the BF test statistic in (4) obtains the value of -2.399 , which is insignificant even at a $10 \%$ level. These results suggest that consumption can be described by a periodically integrated AR(1) process [PIAR(1)]. Nonlinear least squares gives $\hat{\phi}_{1}=1.106, \hat{\phi}_{2}=0.937, \hat{\phi}_{3}=0.998$ and $\hat{\phi}_{4}=$ $1 / \hat{\phi}_{1} \hat{\phi}_{2} \hat{\phi}_{3}=0.967$.

\section{Periodic Integration}

Before we turn to the analysis of the income series along similar lines as above, we take a closer look at a property of a PIAR(1) process. To keep the notation simple, consider again the PAR(1) process $z_{t}=\psi_{s} z_{t-1}+\varepsilon_{t}$, which can be written as $A_{0} Z_{T}=A_{1} Z_{T-1}+\varepsilon_{T}$ with the $A_{0}$ and $A_{1}$ as in (5). The multivariate model can be written as

$$
Z_{T}=A_{0}^{-1} A_{1} Z_{T-1}+A_{0}^{-1} \varepsilon_{T},
$$

i.e. as a standard vector autoregressive process of order 1 [VAR(1)], where 


$$
A_{0}^{-1} A_{1}=\left[\begin{array}{cccc}
0 & 0 & 0 & \psi_{1} \\
0 & 0 & 0 & \psi_{1} \psi_{2} \\
0 & 0 & 0 & \psi_{1} \psi_{2} \psi_{3} \\
0 & 0 & 0 & 1
\end{array}\right]
$$

given the restriction that $\psi_{1} \psi_{2} \psi_{3} \psi_{4}=1$. Notice that $A_{0}^{-1} A_{1}$ is an idempotent matrix, i.e. $\left(A_{0}^{-1} A_{1}\right)^{m}=A_{0}^{-1} A_{1}$. By recursively substituting lagged $Z_{T}$ variables in (8) until $T=0$, one obtains the following expression for $Z_{T}$ :

$$
Z_{T}=A_{0}^{-1} A_{1} Z_{0}+A_{0}^{-1} \varepsilon_{T}+A_{0}^{-1} A_{1} A_{0}^{-1} \sum_{i=1}^{T-1} \varepsilon_{i},
$$

where

$$
A_{0}^{-1} A_{1} A_{0}^{-1}=\left[\begin{array}{cccc}
1 & \psi_{1} \psi_{3} \psi_{4} & \psi_{1} \psi_{4} & \psi_{1} \\
\psi_{2} & 1 & \psi_{1} \psi_{2} \psi_{4} & \psi_{1} \psi_{2} \\
\psi_{2} \psi_{3} & \psi_{3} & 1 & \psi_{1} \psi_{2} \psi_{3} \\
\psi_{2} \psi_{3} \psi_{4} & \psi_{3} \psi_{4} & \psi_{4} & 1
\end{array}\right],
$$

and $\sum_{i=1}^{T-1} \varepsilon_{i}$ is a $(4 \times 1)$ vector containing the accumulation of shocks in the four seasons, i.e. $\sum_{i=1}^{T-1} \varepsilon_{i}=\left(\sum_{i=1}^{T-1} \varepsilon_{1 i}, \sum_{i=1}^{T-1} \varepsilon_{2 i}, \sum_{i=1}^{T-1} \varepsilon_{3 i}, \sum_{i=1}^{T-1} \varepsilon_{4 i}\right)^{\prime}$. Given that the model has periodically varying parameters, i.e. that not all entries of (11) are equal to 1 , it is clear from (10) with (11) that the impact of the accumulation of shocks varies with the season. Note that when all $\psi_{s}$ are equal to 1 , this impact of shocks is the same across all seasons, and then the first order model reduces to the familiar expression: $z_{t}=z_{0}+\sum_{i=1}^{t} \varepsilon_{i}$. In other words, since the impact of the shocks varies with the season, as can be seen from (11), a periodically integrated time series model implies that the stochastic trend effects the "seasonal" pattern, i.e. the differences between $Z_{s T}$ and $Z_{s-1, T}$ for $s=1,2,3,4$ are not constant over time because of the stochastic trend pattern. Hence, the stochastic trend and the seasonal pattern are not independent when a time series is periodically integrated. This implication of PI processes may complicate cointegration analysis, and in the next section we will propose and evaluate cointegration testing methods that can take account of this relationship between seasons and stochastic trend.

\section{Further Empirical Results}

Before we consider cointegration analysis between consumption and income, we report some further empirical results.

The first results concern the consumption series again. Considering the graphs in figure 2, one may also conjecture that the changes in the seasonal pattern can be caused by deterministic changes instead of the stochastic trend. In other 
words, one may hypothesize that $c_{t}$ is not generated by a PIAR(1) model as we have found above, but that it is generated by a model like

$$
\Delta_{1} c_{t}=\lambda_{s}+\lambda_{s}^{*} D U M_{t \geq 74.1}+\lambda_{s}^{* *} D U M_{t \geq 79.1}+\tau_{t},
$$

where $D U M_{t \geq 74.1}$ and $D U M_{t \geq 79.1}$ are $(0, \ldots, 0,1,1, \ldots, 1)$ dummy variables with the first value of 1 when $t=74.1$ and 79.1, respectively, and where $\lambda_{s}$ are four constant seasonal means, the parameters of which change from 1974.1 onwards into $\lambda_{s}+\lambda_{s}^{*}$ and from 1979.1 onwards into $\lambda_{s}+\lambda_{s}^{*}+\lambda_{s}^{* *}$. One may now compare (7) and (12) versus a model that nests both models using $F$ tests. This general model considers a regression of $\Delta_{1} c_{t}$ on $\left(\phi_{s}-1\right) c_{t}$ and the 12 dummy variables in (12) under the restriction that $\phi_{1} \phi_{2} \phi_{3} \phi_{4}=1$.The $F(3,94)$ test for the restrictions implied by the model in (12), i.e. all $\left(\phi_{s}-1\right)=0$, obtains a value of 10.246 , which is significant at the $1 \%$ level. The $F(8,94)$ test for the restrictions implied by the PIAR model in (7) obtains the insignificant value of 1.352. Hence, the model in (12) can be rejected versus the model in (7), and we conclude that a PIAR(1) model gives an adequate description of the consumption variable.

Finally, along similar lines as we construct model (7), we find that a PAR(1) is also appropriate for the income variable. Ordinary least squares gives

$$
y_{t}=\hat{\delta}_{s}+\hat{\alpha}_{s} y_{t-1}+\hat{v}_{t},
$$

with

$$
\begin{array}{cccc}
\hat{\delta}_{1}=-0.120 & \hat{\delta}_{2}=0.371 & \hat{\delta}_{3}=0.417 & \hat{\delta}_{4}=-0.253 \\
(0.076) & (0.069) & (0.072) & (0.077) \\
\hat{\alpha}_{1}=1.001 & \hat{\alpha}_{2}=0.955 & \hat{\alpha}_{3}=0.950 & \hat{\alpha}_{4}=1.047 \\
(0.009) & (0.009) & (0.009) & (0.010)
\end{array}
$$

A regression of $\hat{v}_{t}^{2}$ on a constant and three seasonal dummies obtains an $F$ statistic of 1.138 , implying that no seasonal heteroskedastic error process has to be included in (13). The $F$ test for the hypothesis $\alpha_{s}=\alpha$ obtains a value of 23.738, and this hypothesis is rejected at any reasonable significance level. Even if we include $\omega_{2} y_{t-2}, \ldots, \omega_{8} y_{t-8}$ in the PAR(1) model, where $\omega_{2}$ through $\omega_{8}$ are nonperiodic parameters, the $F$ test statistic for the hypothesis $\alpha_{s}=\alpha$ has a value of 6.653 , which is still significant at the $1 \%$ level. The value of the product $\hat{\alpha}_{1} \hat{\alpha}_{2} \hat{\alpha}_{3} \hat{\alpha}_{4}$ in (13) is 0.951 , and the test for the hypothesis that this product is equal to one yields an insignificant $B F$ test statistic value of -2.849 . Again, the hypothesis of a unit root cannot be rejected, implying that income can also be described by a PIAR(1) process. Note that the rejection of the hypothesis $\alpha_{s}=$ $\alpha$ in a PAR(1) process automatically implies that, as is the case for $c_{t}$, the $\Delta_{1}$ filter for $y_{t}$ is not appropriate. This could already be seen from the graphs in figure 3 , where the $\Delta_{1}$ transformed time series appear to be trending, suggesting that $\Delta_{1}$ is not the appropriate filter to remove the stochastic trend. Finally, the application of nonlinear least squares gives $\hat{\alpha}_{1}=1.014, \hat{\alpha}_{2}=0.966, \hat{\alpha}_{3}=0.963$ and $\hat{\alpha}_{4}=1 / \hat{\alpha}_{1} \hat{\alpha}_{2} \hat{\alpha}_{3}=1.060$. 
To summarize, the empirical results reported in this section for the univariate time series for German consumption and income for the period 1960.1-1987.4 suggest that both series are periodically inegrated. This means that the stochastic trend in each of the series has an impact on the seasonal fluctuations, and hence that trend and seasons are not independent. An implication of this result is that tests for cointegration should take account of this relationship. In the next section we propose, evaluate and apply several useful cointegration testing methods which satisfy this property.

\section{Bivariate Analysịs}

In this section we investigate several methods that can be useful to test for cointegration between two periodically integrated univariate time series. First, we discuss a simple cointegration method in case two series can be described by simple PAR(1) models. This method is, however, not very useful for higher order PAR models, and therefore we propose two alternative methods. The first step in these methods involves the extraction of the stochastic trend from each of the series, while in the second step the two stochastic trends are compared using familiar cointegration techniques. Given that we find only a single unit root in the univariate series, we do not face any identification problems with respect to the extraction of the stochastic trend. The fourth cointegration testing method we propose and apply in this section amounts to an extension to periodic VAR models of the Johansen maximum likelihood method. We choose the notation $C_{T}$ and $Y_{T}$ for the $(4 \times 1)$ vector series containing the annual observations of consumption and income in each of the seasons, i.e. $C_{T}=\left(C_{1 T}, C_{2 T}, C_{3 T}, C_{4 T}\right)^{\prime}$ and $Y_{T}=\left(Y_{1 T}, Y_{2 T}, Y_{3 T}, Y_{4 T}\right)^{\prime}$, where $C_{s T}$ and $Y_{s T}$ denote the observations on consumption and income in quarter $s$ in year $T$, respectively.

Stochastic Trends in a PIAR(1) Process

For a periodically integrated AR(1) process, the application of theorem 4.1 in Johansen (1991) yields some simple results. Consider again the PAR(1) model as in (5) and (6), i.e. $z_{t}=\psi_{s} z_{t-1}+\varepsilon_{t}$. Given (8), one can write

$$
\Delta Z_{T}=\left(A_{0}^{-1} A_{1}-I_{4}\right) Z_{T-1}+A_{0}^{-1} \varepsilon_{T},
$$

where $\Delta$ denotes the first order differencing filter for annual time series, and where $I_{4}$ is the 4-dimensional identity matrix. Given (9) it is easy to see that the 
matrix $\left(A_{0}^{-1} A_{1}-I_{4}\right)$ can be decomposed as $\gamma \beta^{\prime}$, see Johansen (1991), with

$$
\gamma=\left[\begin{array}{rrr}
-1 & 0 & 0 \\
0 & -1 & 0 \\
0 & 0 & -1 \\
0 & 0 & 0
\end{array}\right] \text { and } \beta=\left[\begin{array}{ccc}
1 & 0 & 0 \\
0 & 1 & 0 \\
0 & 0 & 1 \\
-\psi_{1} & -\psi_{1} \psi_{2} & -\psi_{1} \psi_{2} \psi_{3}
\end{array}\right] \text {, }
$$

such that $\gamma_{\perp}=(0,0,0,1)^{\prime}$. An application of theorem 4.1 in Johansen (1991) results in

$$
Z_{T}=\beta_{\perp}\left(\gamma_{\perp}^{\prime} \beta_{\perp}\right)^{-1} \gamma_{\perp}^{\prime} A_{0}^{-1} S_{T}+C(B) \varepsilon_{T},
$$

where $S_{T}=\sum_{j=1}^{T} \varepsilon_{j}$ and $C(B)$ is an invertible matrix lag polynomial. Given the presence of a single unit root in the $Z_{T}$ process, the common stochastic trend in $Z_{T}$ is now simply equal to $\gamma_{\perp}^{\prime} A_{0}^{-1} S_{T}$. Since

$$
A_{0}^{-1}=\left[\begin{array}{cccc}
1 & 0 & 0 & 0 \\
\psi_{2} & 1 & 0 & 0 \\
\psi_{2} \psi_{3} & \psi_{3} & 1 & 0 \\
\psi_{2} \psi_{3} \psi_{4} & \psi_{3} \psi_{4} & \psi_{4} & 1
\end{array}\right]
$$

we can write

$$
\gamma_{\perp}^{\prime} A_{0}^{-1} S_{T}=\left(\psi_{2} \psi_{3} \psi_{4}, \psi_{3} \psi_{4}, \psi_{4}, 1\right) \sum_{j=1}^{T}\left[\begin{array}{c}
Z_{1 j}-\psi_{1} Z_{4, j-1} \\
Z_{2 j}-\psi_{2} Z_{1 j} \\
Z_{3 j}-\psi_{3} Z_{2 j} \\
Z_{4 j}-\psi_{4} Z_{3 j}
\end{array}\right]=Z_{4 T},
$$

conditional on suitable starting values and on the restriction $\psi_{1} \psi_{2} \psi_{3} \psi_{4}=1$. The result in (16) implies that each $Z_{s T}$ series can be a common trend.

As a second step in our case of the consumption and income processes $C_{T}$ and $Y_{T}$, one may compare the annual time series $C_{s T}$ and $Y_{s T}$ in cointegration exercises. We obtain Dickey-Fuller test values (no constant, no lags) for the residuals of the regressions of $C_{s T}$ on a constant and $Y_{s T}, s=1,2,3,4$, of $-3.702,-2.818,-1.655$ and -1.462 , respectively. Comparing these values with the fractiles in MacKinnon (1991), there seems to be cointegration at a $5 \%$ level in the first quarter only.

For periodically integrated $\operatorname{AR}(p)$ processes, where $p$ exceeds 1 , the expressions similar to (15) and (16) become much more complicated. Hence, in case of such higher order processes, one may want to use simpler methods to extract the stochastic trends from each of the series.

\section{Extracting Stochastic Trends Using the Box-Tiao Method}

One simple approach to extract a stochastic trend from the $(4 \times 1)$ vector series $C_{T}$ and $Y_{T}$ is to use the Box and Tiao (1977) [BT] method. For a vector 
process of order 1, as in (8), this method considers the eigenvalue problem

$$
\left|\lambda I-\left(X_{T}^{\prime} X_{T}\right)^{-1} X_{T}^{\prime} X_{T-1}\left(X_{T-1}^{\prime} X_{T-1}\right)^{-1} X_{T-1}^{\prime} X_{T}\right|=0
$$

where $X_{T}$ is $C_{T}$ or $Y_{T}$ when corrected for their respective means. The eigenvector which corresponds to the largest eigenvalue is the most nonstationary linear combination that can be constructed from the four $X_{s T}$ series, see Box and Tiao (1977) for details. One can consider this linear combination to be the stochastic trend in the multivariate system for $X_{T}$. Denoting these stochastic trends for $C_{T}$ and $Y_{T}$ as $C_{T}^{B T}$ and $Y_{T}^{B T}$, one can compare these annually observed variables in a second step of cointegration analysis.

The obvious question is now which critical values to use for the test statistics in a second cointegration testing step. In tables 1 through 4 we report on the empirical fractiles and the empirical size of the Engle-Granger (1987) [EG] and the Johansen-Juselius (1990) [JJ] cointegration testing methods, after we have extracted the stochastic trend using the BT method. The EG method amounts to calculating the Durbin-Watson statistic [CRDW] and the (augmented) Dickey-Fuller test [CRDF] for the cointegrating regression of $C_{T}^{B T}$ on a constant and $Y_{T}^{B T}$. The standard critical values are taken from MacKinnon (1991) for 25 observations. The $\mathrm{JJ}$ method amounts to regressing $\Delta C_{T}^{B T}, \Delta Y_{T}^{B T}$, $C_{T-1}^{B T}$ and $Y_{T-1}^{B T}$ on a constant and lagged $\Delta C_{T}^{B T}$ and $\Delta Y_{T}^{B T}$ variables, giving the $(2 \times 1)$ vectors of residuals $R_{0 T}$ and $R_{1 T}$ and the residual product matrices

$$
S_{i j}=(1 / N) \sum_{T=1}^{N} R_{i T} R_{j T}^{\prime}, \text { for } i, j=0,1 .
$$

The next step is to solve the eigenvalue problem

$$
\left|\lambda S_{11}-S_{10} S_{00}^{-1} S_{01}\right|=0 \text {, }
$$

which gives the eigenvalues $\hat{\lambda}_{1}>\hat{\lambda}_{2}$ and the corresponding eigenvectors $\hat{v}_{1}$ and $\hat{v}_{2}$, in our case of only two variables. The two test statistics to check for the presence of cointegration are the maximal eigenvalue test and the trace test, see Johansen and Juselius (1990) for details. For the case of two series of length 25 , we generate the critical values for the case of two independent random walks and display the results in table A1 in the appendix. These fractiles will be used to evaluate the fractiles of the $\mathrm{JJ}$ tests in case the tests are considered for estimated stochastic trends from PIAR models.

The first panel in the tables 1 through 4 displays the empirical size and fractiles of the CRDW and CRDF as well as of the JJ-trace test, to be denoted as $\operatorname{Tr}(r \leq 1)$ and $\operatorname{Tr}(r \leq 0)$, where $r$ is the number of cointegrating relationships between $C_{T}^{B T}$ and $Y_{T}^{B T}$. The results for the maximal eigenvalue test are very similar, and are not reported to save space. The data generating processes [DGP] in the tables 1 through 4 consists of two independent PIAR(1) processes. We have selected four sets of DGPs in order to investigate whether the choice for certain parameter values effects the Monte Carlo outcomes. The DGP in table 4 corresponds to the estimated univariate models for consump- 
Table 1. Empirical performance of cointegration tests (size) in case the DGP consists of two independent PIAR(1) processes. DGP is $y_{t}=\phi_{s} y_{t-1}+\varepsilon_{t}$ and $x_{t}=\alpha_{s} x_{t-1}+v_{t}$, where $\varepsilon_{t}, v_{t}$ are $N(0,1)$ and $\phi_{1}=\alpha_{1}=1.25, \phi_{2}=\alpha_{2}=0.8, \phi_{3}=\alpha_{3}=2.0, \phi_{4}=\alpha_{4}=0.5$. Based on 5000 replications of effective sample size $n=100$ or $N=25$

\begin{tabular}{llrrrrrr}
\hline Method $^{(1)}$ & \multicolumn{3}{c}{ Empirical fractiles } & \multicolumn{4}{c}{ Empirical size $^{(2)}$} \\
& & \multicolumn{1}{c}{$5 \%$} & \multicolumn{1}{c}{$10 \%$} & \multicolumn{1}{c}{$\mathbf{2 0} \%$} & $5 \%$ & $10 \%$ & $20 \%$ \\
\hline EG after BT & CRDW & 1.33 & 1.15 & 0.93 & 6.28 & 12.26 & 21.70 \\
& CRDF & -3.74 & -3.35 & -2.88 & 5.90 & 11.92 & 22.10 \\
JJ after BT & $\operatorname{Tr}(r \leq 1)$ & 8.76 & 6.97 & 5.19 & 6.10 & 11.10 & 21.88 \\
& $\operatorname{Tr}(r \leq 0)$ & 19.46 & 17.23 & 14.29 & 6.20 & 11.76 & 21.44 \\
\hline EG after GG & CRDW & 1.12 & 0.96 & 0.79 & 2.28 & 5.72 & 12.88 \\
& CRDF & -3.27 & -2.96 & -2.60 & 2.04 & 5.50 & 13.96 \\
& CRDF after GG & -3.45 & -3.11 & -2.72 & 3.54 & 7.80 & 17.60 \\
& $\operatorname{Tr}(r \leq 1)$ & 6.89 & 5.78 & 4.44 & 2.20 & 5.62 & 15.78 \\
& $\operatorname{Tr}(r \leq 0)$ & 16.94 & 14.79 & 12.56 & 2.26 & 5.44 & 13.30 \\
& $\operatorname{Tr}(r \leq 1)^{(3)}$ & 8.18 & 6.65 & 5.00 & 4.76 & 9.76 & 20.68 \\
& $\operatorname{Tr}(r \leq 0)^{(3)}$ & 20.25 & 17.66 & 14.93 & 7.14 & 13.50 & 24.56 \\
\hline Periodic JJ & $\operatorname{Tr}(r \leq 1)$ & 8.30 & 6.75 & 5.03 & 5.10 & 10.34 & 20.64 \\
& $\operatorname{Tr}(r \leq 0)$ & 19.06 & 16.70 & 14.24 & 5.28 & 10.50 & 21.46 \\
\hline
\end{tabular}

(1) The methods and abbreviations are given in the text in section 3. Unless otherwise indicated, the CRDF statistics are based on an auxiliary regression that contains no constant and no lags, and the JJ-trace test statistics are calculated for a VAR(1) process.

${ }^{(2)}$ In the empirical size columns we report on the rejection frequency when the standard critical values for nonperiodic models are used, which are given in Mackinnon (1991) and table A1 in the appendix.

(3) For the CRDF statistic, the auxiliary regression contains no constant and one lag. The trace test statistics are calculated for a VAR(2) process of $C_{T}^{G G}$ and $Y_{T}^{G G}$.

tion and income in the previous section. As can be observed from the numbers in the first panel of all four tables, it seems that empirical rejection frequencies are very close to the nominal sizes even for a sample size of 100 quarterly, i.e. 25 annual, observations. Hence, we may use the standard critical values in our cointegration analysis of $C_{T}^{B T}$ and $Y_{T}^{B T}$. However, since we have generated the critical values in case the DGP consists of PIAR(1) models with parameters as those in the previous section, we will use the critical values displayed in the first panel of table 4 in the next empirical analysis.

An application of the Box-Tiao method in (17) to extract the stochastic trend from the $C_{T}$ series gives the four eigenvalues are $0.999,0.303,0.180$ and 0.103 , while for $Y_{T}$ income they are $0.998,0.216,0.117$ and 0.094 . It is clear from these eigenvalues that there seems to be only a single unit root in the periodic models. This confirms the results of the application of the BF test in (4). The most nonstationary linear combinations are

$$
C_{T}^{B T}=1.000 C_{1 T}+0.250 C_{2 T}+0.032 C_{3 T}-0.112 C_{4 T}
$$

and 
Table 2. Empirical performance of cointegration tests (size) in case the DGP consists of two independent PIAR(1) processes. DGP is $y_{t}=\phi_{s} y_{t-1}+\varepsilon_{t}$ and $x_{t}=\alpha_{s} x_{t-1}+v_{t}$, where $\varepsilon_{t}, v_{t}$ are $N(0,1)$ and $\phi_{1}=1.25, \phi_{2}=0.8, \phi_{3}=2.0, \phi_{4}=0.5, \alpha_{1}=\alpha_{2}=\alpha_{3}=\alpha_{4}=1$ Based on 5000 replications of effective sample size $n=100$ or $N=25$

\begin{tabular}{|c|c|c|c|c|c|c|c|}
\hline \multirow[t]{2}{*}{ Method $^{(1)}$} & & \multicolumn{3}{|c|}{ Empirical fractiles } & \multicolumn{3}{|c|}{ Empirical size ${ }^{(2)}$} \\
\hline & & $5 \%$ & $10 \%$ & $20 \%$ & $5 \%$ & $10 \%$ & $20 \%$ \\
\hline \multirow[t]{2}{*}{ EG after BT } & CRDW & 1.34 & 1.15 & 0.93 & 6.32 & 12.34 & 22.04 \\
\hline & CRDF & -3.70 & -3.33 & -2.89 & 5.78 & 11.70 & 22.38 \\
\hline \multirow[t]{2}{*}{$\mathrm{J} \mathbf{J}$ after $\mathbf{B T}$} & $\operatorname{Tr}(r \leq 1)$ & 8.57 & 6.80 & 5.04 & 5.66 & 10.24 & 20.84 \\
\hline & $\operatorname{Tr}(r \leq 0)$ & 19.04 & 16.74 & 14.20 & 5.24 & 10.48 & 21.14 \\
\hline \multirow[t]{3}{*}{ EG after GG } & CRDW & 1.12 & 0.96 & 0.80 & 2.40 & 5.90 & 13.30 \\
\hline & CRDF & -3.31 & -2.97 & -2.60 & 2.44 & 5.90 & 13.70 \\
\hline & $\mathrm{CRDF}^{(3)}$ & -3.57 & -3.19 & -2.76 & 4.46 & 9.20 & 18.68 \\
\hline \multirow[t]{4}{*}{$J J$ after $G G$} & $\operatorname{Tr}(r \leq 1)$ & 6.85 & 5.65 & 4.35 & 1.84 & 5.50 & 14.68 \\
\hline & $\operatorname{Tr}(r \leq 0)$ & 16.88 & 14.78 & 12.50 & 2.56 & 5.50 & 12.78 \\
\hline & $\operatorname{Tr}(r \leq 1)^{(3)}$ & 8.29 & 6.61 & 5.01 & 5.02 & 9.50 & 20.88 \\
\hline & $\operatorname{Tr}(r \leq 0)^{(3)}$ & 19.98 & 17.78 & 15.07 & 7.32 & 13.90 & 24.76 \\
\hline \multirow[t]{2}{*}{ Periodic JJ } & $\operatorname{Tr}(r \leq 1)$ & 8.25 & 6.70 & 4.98 & 4.96 & 10.02 & 20.48 \\
\hline & $\operatorname{Tr}(r \leq 0)$ & 18.35 & 16.18 & 13.56 & 4.22 & 9.10 & 18.02 \\
\hline
\end{tabular}

(1) The methods and abbreviations are given in the text in section 3. Unless otherwise indicated, the CRDF statistics are based on an auxiliary regression that contains no constant and no lags, and the JJ-trace test statistics are calculated for a VAR(1) process.

${ }^{(2)}$ In the empirical size columns we report on the rejection frequency when the standard critical values for nonperiodic models are used, which are given in Mackinnon (1991) and table A1 in the appendix.

(3) For the CRDF statistic, the auxiliary regression contains no constant and one lag. The trace test statistics are calculated for a VAR(2) process of $C_{T}^{G G}$ and $Y_{T}^{G G}$.

$$
Y_{T}^{B T}=1.000 Y_{1 T}-0.147 Y_{2 T}+0.550 Y_{3 T}-0.350 Y_{4 T} .
$$

Given that the measurement units are the same across the $C_{s T}$ and $Y_{s T}$ series, one can conclude from these expressions that the first quarter contributes the most to the stochastic trend for consumption as well as for income. Graphs of the two common trend series are given in figure 4.

An Engle and Granger (1987) type of cointegrating regression yields

$$
\begin{gathered}
C_{T}^{B T}=-0.170+1.114 Y_{T}^{B T}, \\
(0.116)(0.014)
\end{gathered}
$$

with $\mathrm{CRDW}=0.955$ and $\mathrm{CRDF}=-3.431$. Comparing these values with the fractiles in table 4 , it can be seen that the CRDW value of 0.955 is only significant at a $20 \%$ level, while the CRDF value of -3.431 is significant at a $10 \%$ level. An application of the Johansen-Juselius method to a VAR(1) for the $C_{T}^{B T}$ and $Y_{T}^{B T}$ series gives the trace statistic values $\operatorname{Tr}(r \leq 1)=6.388$ and $\operatorname{Tr}(r \leq 0)=$ 19.584. It is clear from the empirical fractiles in table 4 that the latter statistic is significant at a $5 \%$ level. Hence, the $\mathrm{JJ}$ method seems to indicate that there is 
Table 3. Empirical performance of cointegration tests (size) in case the DGP consists of two independent PIAR(1) processes. DGP is $y_{t}=\phi_{s} y_{t-1}+\varepsilon_{t}$ and $x_{t}=\alpha_{s} x_{t-1}+v_{t}$, where $\varepsilon_{t}, v_{t}$ are $N(0,1)$ and $\phi_{1}=\alpha_{1}=1.105, \phi_{2}=\alpha_{2}=0.937, \phi_{3}=\alpha_{3}=0.998, \phi_{4}=\alpha_{4}=0.968$ Based on 5000 replications of effective sample size $n=100$ or $N=25$

\begin{tabular}{|c|c|c|c|c|c|c|c|}
\hline \multirow[t]{2}{*}{ Method $^{(1)}$} & & \multicolumn{3}{|c|}{ Empirical fractiles } & \multicolumn{3}{|c|}{ Empirical size ${ }^{(2)}$} \\
\hline & & $5 \%$ & $10 \%$ & $20 \%$ & $5 \%$ & $10 \%$ & $20 \%$ \\
\hline \multirow[t]{2}{*}{ EG after BT } & CRDW & 1.31 & 1.11 & 0.90 & 7.72 & 10.50 & 20.08 \\
\hline & CRDF & -3.64 & -3.28 & -2.81 & 5.16 & 10.54 & 20.06 \\
\hline \multirow[t]{2}{*}{$\mathrm{JJ}$ after BT } & $\operatorname{Tr}(r \leq 1)$ & 8.52 & 6.85 & 5.09 & 5.64 & 10.56 & 21.50 \\
\hline & $\operatorname{Tr}(r \leq 0)$ & 19.28 & 16.67 & 14.13 & 5.66 & 10.36 & 20.94 \\
\hline \multirow[t]{3}{*}{ EG after GG } & CRDW & 1.14 & 0.97 & 0.80 & 2.74 & 6.26 & 13.42 \\
\hline & CRDF & -3.33 & -2.99 & -2.61 & 2.34 & 6.18 & 14.26 \\
\hline & $\mathrm{CRDF}^{(3)}$ & -3.54 & -3.19 & -2.79 & 4.42 & 9.34 & 19.50 \\
\hline \multirow[t]{4}{*}{$\mathbf{J} \mathbf{J}$ after $\mathbf{G G}$} & $\operatorname{Tr}(r \leq 1)$ & 6.88 & 5.68 & 4.34 & 2.36 & 5.64 & 14.70 \\
\hline & $\operatorname{Tr}(r \leq 0)$ & 16.90 & 14.94 & 12.48 & 2.58 & 5.40 & 13.26 \\
\hline & $\operatorname{Tr}(r \leq 1)^{(3)}$ & 8.16 & 6.61 & 5.00 & 4.74 & 9.76 & 20.72 \\
\hline & $\operatorname{Tr}(r \leq 0)^{(3)}$ & 20.64 & 17.93 & 15.02 & 8.02 & 14.36 & 25.20 \\
\hline \multirow[t]{2}{*}{ Periodic JJ } & $\operatorname{Tr}(r \leq 1)$ & 8.26 & 6.66 & 4.98 & 5.00 & 9.84 & 20.44 \\
\hline & $\operatorname{Tr}(r \leq 0)$ & 18.73 & 16.43 & 13.86 & 4.60 & 9.64 & 19.44 \\
\hline
\end{tabular}

(1) The methods and abbreviations are given in the text in section 3. Unless otherwise indicated, the CRDF statistics are based on an auxiliary regression that contains no constant and no lags, and the JJ-trace test statistics are calculated for a VAR(1) process.

${ }^{(2)}$ In the empirical size columns we report on the rejection frequency when the standard critical values for nonperiodic models are used, which are given in MacKinnon (1991) and table A1 in the appendix.

(3) For the CRDF statistic, the auxiliary regression contains no constant and one lag. The trace test statistics are calculated for a VAR(2) process of $C_{T}^{G G}$ and $Y_{T}^{G G}$.

cointegration between the stochastic trends extracted using the BT method. According to the $\mathrm{JJ}$ method, the cointegrating vector is $(1,-1.114)$, which is equal to the EG outcome in (22). Using (20) and (21) the cointegration relation between $C_{T}$ and $Y_{T}$ is

$$
\begin{aligned}
& C_{1 T}-1.11 Y_{1 T}+0.25 C_{2 T}+0.16 Y_{2 T}+0.03 C_{3 T}-0.61 Y_{3 T} \\
& \quad-0.11 C_{4 T}+0.39 Y_{4 T}
\end{aligned}
$$

It is clear that this relation is dominated by its $C_{1 T}-1.11 Y_{1 T}$ component, i.e. a likely cointegration relation between $C_{T}$ and $Y_{T}$ in the first quarter. This outcome closely corresponds to the finding earlier in this section.

\section{Extracting Stochastic Trends Using the Gonzalo-Granger Method}

The Gonzalo-Granger (1994) method to extract the stochastic trend exploits the duality between cointegration and stochastic trends. For our $X_{T}$ series, 
Table 4. Empirical performance of cointegration tests (size) in case the DGP consists of two independent PIAR(1) processes. DGP is $y_{t}=\phi_{s} y_{t-1}+\varepsilon_{t}$ and $x_{t}=\alpha_{s} x_{t-1}+v_{t}$, where $\varepsilon_{t}, v_{t}$ are $N(0,1)$ and $\phi_{1}=1.106, \phi_{2}=0.937, \phi_{3}=0.998, \phi_{4}=0.967, \alpha_{1}=1.014, \alpha_{2}=0.966, \alpha_{3}=0.963, \alpha_{4}=1.060$. Based on 5000 replications of effective sample size $n=100$ or $N=25$

\begin{tabular}{llrrrrrr}
\hline Method $^{(1)}$ & & \multicolumn{3}{c}{ Empirical fractiles } & \multicolumn{3}{c}{ Empirical size ${ }^{(2)}$} \\
& & \multicolumn{1}{c}{$5 \%$} & \multicolumn{1}{c}{$10 \%$} & \multicolumn{1}{c}{$20 \%$} & $5 \%$ & \multicolumn{1}{c}{$5 \%$} & $20 \%$ \\
\hline EG after BT & CRDW & 1.32 & 1.12 & 0.92 & 5.74 & 11.40 & 20.98 \\
& CRDF & -3.75 & -3.32 & -2.87 & 5.82 & 11.20 & 22.88 \\
JJ after BT & $\operatorname{Tr}(r \leq 1)$ & 8.64 & 6.86 & 5.07 & 5.68 & 10.64 & 21.04 \\
& $\operatorname{Tr}(r \leq 0)$ & 19.36 & 16.85 & 14.14 & 5.72 & 10.74 & 20.66 \\
\hline EG after GG & CRDW & 1.12 & 0.98 & 0.81 & 2.28 & 5.66 & 13.82 \\
& CRDF & -3.29 & -2.96 & -2.62 & 2.10 & 5.54 & 13.88 \\
& CRDF ${ }^{(3)}$ & -3.54 & -3.20 & -2.78 & 4.16 & 9.24 & 19.26 \\
& $\operatorname{Tr}(r \leq 1)$ & 6.71 & 5.58 & 4.32 & 1.86 & 5.04 & 14.44 \\
& $\operatorname{Tr}(r \leq 0)$ & 17.09 & 15.02 & 12.53 & 2.86 & 5.96 & 13.14 \\
& $\operatorname{Tr}(r \leq 1)^{(3)}$ & 7.99 & 6.51 & 4.91 & 4.26 & 9.20 & 19.82 \\
& $\operatorname{Tr}(r \leq 0)^{(3)}$ & 20.74 & 18.01 & 15.22 & 8.08 & 14.69 & 25.70 \\
\hline Periodic JJ & $\operatorname{Tr}(r \leq 1)$ & 8.17 & 6.43 & 4.81 & 4.84 & 8.98 & 19.06 \\
& $\operatorname{Tr}(r \leq 0)$ & 19.12 & 16.56 & 13.73 & 5.34 & 9.98 & 18.64 \\
\hline
\end{tabular}

(1) The methods and abbreviations are given in the text in section 3. Unless otherwise indicated, the CRDF statistics are based on an auxiliary regression that contains no constant and no lags, and the JJ-trace test statistics are calculated for a VAR(1) process.

(2) In the empirical size columns we report on the rejection frequency when the standard critical values for nonperiodic models are used, which are given in MacKinnon (1991) and table A1 in the appendix.

(3) For the CRDF statistic, the auxiliary regression contains no constant and one lag. The trace test statistics are calculated for a VAR(2) process of $C_{T}^{G G}$ and $Y_{T}^{G G}$.

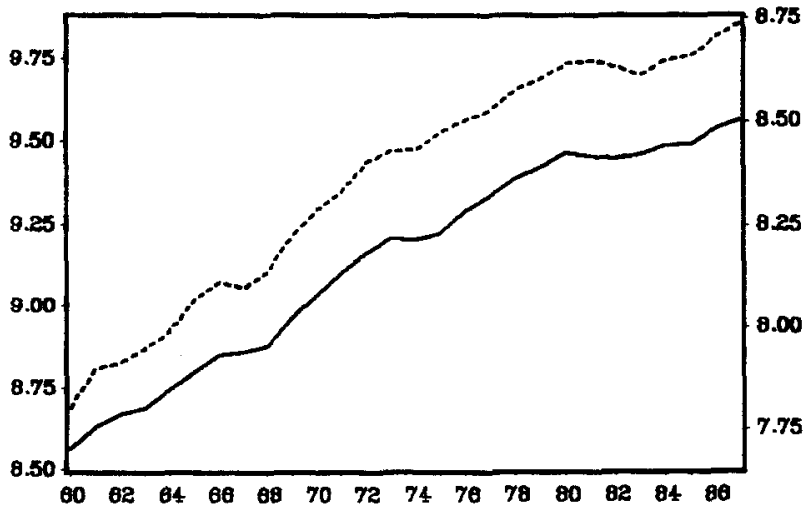

CET -... TET

Fig. 4. Stochastic trends using Box-Tiao method 
where $X_{T}$ is either $C_{T}$ or $Y_{T}$, the procedure is as follows. First one regresses $\Delta X_{T}$ and $X_{T-1}$ on a constant and lagged $\Delta X_{T}$ variables. This gives the $(4 \times 1)$ residual vectors $R_{0 T}$ and $R_{1 T}$ and the $(4 \times 4)$ residual product matrices $S_{i j}, i$, $j=0,1$, calculated in the same way as (18). The next step is to solve the eigenvalue problem $\left|\lambda S_{00}-S_{01} S_{11}^{-1} S_{10}\right|=0$, which gives the eigenvalues $\hat{\lambda}_{1}>\cdots>$ $\hat{\lambda}_{4}$ and eigenvectors $m_{1}, \ldots, m_{4}$. Given that we know that there are three cointegrating relations between the elements of $X_{T}$ for both consumption and income, the stochastic trends here are found to be equal to $m_{4}^{\prime} X_{T}$. We denote these trends extracted using this GG method as $C_{T}^{G G}$ and $Y_{T}^{G G}$.

Again there is the question whether one can use the standard critical values when testing for cointegration between $C_{T}^{G G}$ and $Y_{T}^{G G}$. In the second panel of tables 1 through 4, we report on the empirical fractiles and sizes in case the DGP consists of two independent PIAR(1) processes. In contrast to the outcomes for the BT method, it seems that the empirical rejection frequency is below the nominal size for the CRDW statistic and for the CRDF test in case no lagged variables are included in the auxiliary Dickey-Fuller regression, as well as for the JJ-trace tests in case a VAR(1) for $C_{T}^{G G}$ and $Y_{T}{ }^{G G}$ is assumed to be adequate. However, when we include an additional lag for the CRDF test and consider a VAR(2) instead of a VAR(1) for $C_{T}^{G G}$ and $Y_{T}^{G G}$, the empirical size gets closer to the nominal size. We also perform a similar exercise in case we have 400 quarterly, i.e. 100 annual, observations. The results of these simulations are reported in table 5. It can be observed from the results in this table that the empirical size gets closer to the nominal size in larger samples.

For the empirical analysis below, as well as for the Monte Carlo investigation into the power properties of the various cointegration testing methods proposed in this section, we therefore use the critical values given in MacKinnon (1991), while we add one lag to the Dickey-Fuller regression. Further, we use

Table 5. Empirical performance of cointegration tests (size) in case the DGP consists of two independent PIAR(1) processes. DGP is $y_{t}=\phi_{s} y_{t-1}+\varepsilon_{t}$ and $x_{t}=\alpha_{s} x_{t-1}+v_{t}$, where $\varepsilon_{t}, v_{t}$ are $N(0,1)$ and $\phi_{1}=1.106, \phi_{2}=0.937, \phi_{3}=0.998, \phi_{4}=0.967, \alpha_{1}=1.014, \alpha_{2}=0.966, \alpha_{3}=0.963, \alpha_{4}=1.060$. Based on 5000 replications of effective sample size $n=400$ or $N=100$

\begin{tabular}{llrrrrrr}
\hline Method $^{(1)}$ & & \multicolumn{3}{c}{ Empirical fractiles } & \multicolumn{3}{c}{ Empirical size } \\
& & \multicolumn{1}{c}{$5 \%$} & \multicolumn{1}{c}{$10 \%$} & \multicolumn{1}{c}{$20 \%$} & $5 \%$ & $10 \%$ & $20 \%$ \\
\hline EG after GG & CRDW & 0.36 & 0.30 & 0.24 & 3.38 & 7.62 & 18.82 \\
& CRDF & -3.30 & -3.00 & -2.68 & 4.00 & 9.16 & 18.68 \\
JJ after GG & $\operatorname{Tr}(r \leq 1)$ & 7.79 & 6.34 & 4.79 & 4.22 & 8.74 & 19.22 \\
& $\operatorname{Tr}(r \leq 0)$ & 17.91 & 15.76 & 13.32 & 4.54 & 7.12 & 18.96 \\
\hline
\end{tabular}

(1) The methods and abbreviations are given in the text in section 3. Unless otherwise indicated, the CRDF statistics are based on an auxiliary regression that contains no constant and no lags, and the JJ-trace test statistics are calculated for a VAR(1) process.

(2) In the empirical size columns we report on the rejection frequency when the standard critical values for nonperiodic models are used, which are given in MacKinnon (1991) and table A1 in the appendix. 
Table 6. Empirical performance of cointegration tests (power) in case the bivariate process in (27) and (28) is the DGP Based on 5000 replications of effective sample size $n=100$ or $N=25$

\begin{tabular}{|c|c|c|c|c|}
\hline \multirow[t]{2}{*}{ Method $^{(1)}$} & & \multicolumn{3}{|c|}{ Rejection frequency at nominal size } \\
\hline & & $5 \%$ & $10 \%$ & $20 \%$ \\
\hline \multirow[t]{2}{*}{ EG after BT } & CRDW & 11.26 & 21.54 & 68.68 \\
\hline & CRDF & 7.24 & 14.14 & 27.26 \\
\hline \multicolumn{2}{|l|}{$\mathrm{JJ}$ after $\mathbf{B} \mathrm{T}^{(3)}$} & 49.72 & 57.76 & 60.34 \\
\hline EG after $G^{(2)}$ & CRDF & 9.98 & 19.04 & 34.28 \\
\hline \multicolumn{2}{|l|}{ JJ after $\mathrm{GG}^{(3)(5)}$} & 27.82 & 38.46 & 46.02 \\
\hline \multirow[t]{5}{*}{ Periodic JJ $J^{(4)}$} & Q1 & 49.62 & 57.02 & 58.52 \\
\hline & Q2 & 46.44 & 54.34 & 56.88 \\
\hline & Q3 & 47.40 & 54.84 & 57.08 \\
\hline & Q4 & 49.25 & 56.48 & 57.12 \\
\hline & all $Q$ & 26.38 & 32.58 & 35.76 \\
\hline
\end{tabular}

(1) The methods and abbreviations are given in the text in section 3. Unless otherwise indicated, the CRDF statistics are based on an auxiliary regression that contains no constant and no lags, and the JJ-trace test statistics are calculated for a VAR(1) process.

(2) The CRDW does not have the correct size, and is therefore not used here. The results for the CRDF are based on a Dickey-Fuller regression with one lag.

${ }^{(3)}$ Reject $\operatorname{Tr}(r \leq 0)$ and do not reject $T R(r \leq 1)$.

${ }^{(4)}$ Reject $\operatorname{Tr}(r \leq 0)$ and do not reject $\operatorname{TR}(r \leq 1)$ per quarter $Q_{s}$ and over all the four quarters at the same time.

${ }^{(5)}$ Results based on a VAR(2).

the critical values in table $\mathrm{A} 1$ in the appendix for the $\mathrm{JJ}$-trace test statistics, where we consider a VAR(2) model for $C_{T}^{G G}$ and $Y_{T}^{G G}$ variables.

The application of the GG method to extract the stochastic trend from the $(4 \times 1)$ vector series $C_{T}$ and $Y_{T}$ results in

$$
C_{T}^{G G}=-0.493 C_{1 T}-0.381 C_{2 T}+0.551 C_{3 T}+1.000 C_{4 T}
$$

and

$$
Y_{T}^{G G}=-0.240 Y_{1 T}-0.297 Y_{2 T}+0.038 Y_{3 T}+1.000 Y_{4 T},
$$

Graphs of these two series appear in figure 5.

The Engle-Granger cointegrating regression looks like

$$
\begin{gathered}
C_{T}^{G G}=0.741+1.144 Y_{T}^{G G}, \\
(0.096)(0.023)
\end{gathered}
$$

where CRDW takes a value of 0.962 , and the CRDF with 0 and 1 additional lags has the values -2.690 and -1.585 , respectively. Comparing these values with the standard critical values, we cannot reject the null hypothesis of no cointegration. An application of the JJ method to a VAR(1) and VAR(2) for $C_{T}^{G G}$ and $Y_{T}^{G G}$ results in the trace test statistics $\operatorname{Tr}(r \leq 1)=1.192, \operatorname{Tr}(r \leq 0)=$ 8.705 , and $\operatorname{Tr}(r \leq 1)=0.206, \operatorname{Tr}(r \leq 0)=4.222$, respectively. Hence, the JJ method does not yield evidence in favour of cointegration either. In sum, it seems that the application of the GG method to extract the stochastic trend 

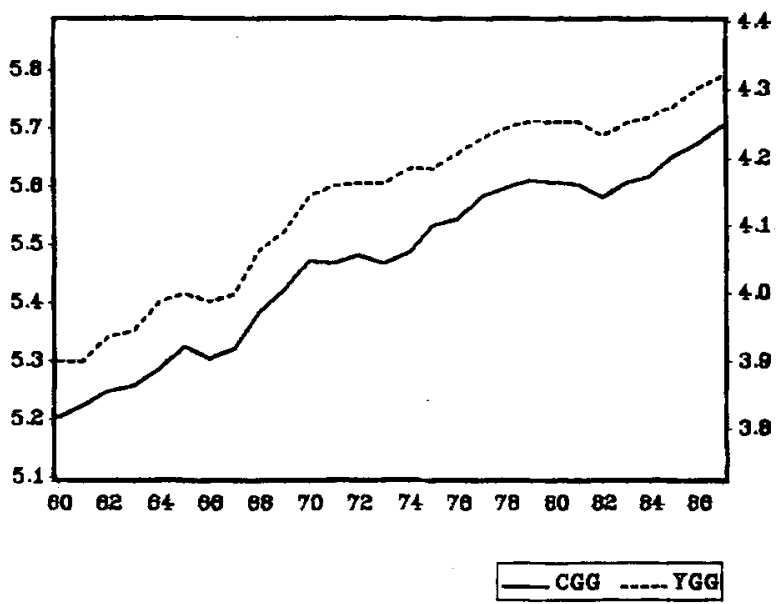

Fig. 5. Stochastic trends using Gonzalo-Granger method

does not yield any evidence for cointegration between consumption and income.

The Johansen Method for Periodic Vector Autoregressions

The final method we propose in this paper which can be useful to test for the presence of cointegration between periodically integrated time series amounts to an extension of the Johansen-Juselius method to periodic VAR models [PVAR]. Given that the differencing filters are $\left(1-\phi_{s} B\right)$ and $\left(1-\alpha_{s} B\right)$ for consumption and income, respectively, one may want to specify for example a PVAR(1) process

$$
\left[\begin{array}{c}
\left(1-\phi_{s} B\right) c_{t} \\
\left(1-\alpha_{s} B\right) y_{t}
\end{array}\right]=\Pi_{s}\left[\begin{array}{l}
c_{t-1} \\
y_{t-1}
\end{array}\right]+\left[\begin{array}{l}
\varepsilon_{t} \\
u_{t_{-}}
\end{array}\right]
$$

which gives four $(2 \times 2) \Pi_{\mathrm{s}}$ matrices, for each of the seasons. The next step may then be to check the rank of each of these matrices, and to investigate whether the $\Pi_{s}$ can be decomposed into $\gamma_{s} \beta_{s}^{\prime}$. A drawback of this method, however, is that the distribution of the JJ-type test statistics will not be a standard distribution like that in table $\mathrm{A} 1$ in the appendix. The intuition behind this conjecture can be observed from considering a rewritten version of the univariate PAR(1) model, i.e.

$$
A_{1} z_{t}=\left(\psi_{s}-1\right) z_{t-1}+\varepsilon_{t}
$$

and assuming that one wants to test the hypothesis $\psi_{s}-1=0$ for all $s$. One may consider testing this hypothesis if one wants to check whether $\Delta_{1}$ is the appropriate differencing filter for a periodic time series. The distribution of a 
Wald test statistic for the hypothesis $\psi_{s}=1$ for all $s$ can be shown to be the sum of a Dickey-Fuller type distribution and a $\chi^{2}(3)$ distribution, see Boswijk and Franses (1994). Hence, this test requires new tables with critical values. Hence, the application of the JJ tests to (24) would also amount to new asymptotic distributions. Using (unreported) simulations we generate critical values for these tests for the case at hand. An application of the $\mathrm{JJ}$ tests results in a similar empirical finding as to be reported below. Furthermore, we also perform a power study (similar as to be reported in Table 6) and do not find that this method outperforms other methods. To summarize, we consider the extension and detailed evaluation of $\mathbf{J J}$ tests useful, although we postpone this analysis for further research.

Alternatively, we propose to apply the Johansen-Juselius cointegration testing approach not to a model like (24), but to each of the seasons separately. The procedure is as follows. One regresses $\left(\Delta C_{s T}, \Delta Y_{s T}\right)$ and $\left(C_{s, T-1}, Y_{s, T-1}\right)$ on a constant and lagged $\Delta C_{s T}$ and $\Delta Y_{s T}$ variables for each of the seasons $s=1,2$, 3 , 4. This gives the $(2 \times 1)$ residual vectors $R_{0 T, s}$ and $R_{1 T, s}$ for each season $s$ and the $(2 \times 2)$ residual product matrices $S_{i j, s}$ constructed as

$$
S_{i j, s}=(1 / N) \sum_{T=1}^{N} R_{i T, s} R_{j T, s}^{\prime}, \text { for } i, j=0,1 \text {. }
$$

The next step is to solve the eigenvalue problem

$$
\left|\lambda S_{11, s}-S_{10, s} S_{00, s}^{-1} S_{01, s}\right|=0,
$$

for each season $s$, which gives the eigenvalues $\hat{\lambda}_{1 s}>\hat{\lambda}_{2 s}$ and the corresponding eigenvectors $\hat{v}_{1 s}$ and $\hat{v}_{2 s}$, in our case of only two variables. One can now apply the trace test statistics $\operatorname{Tr}_{s}(r \leq 1)$ and $\operatorname{Tr}_{s}(r \leq 0)$ to check for the presence of cointegration between $C_{s T}$ and $Y_{s T}$ for each $s$.

Again we check the validity of the critical values in table A1 in the appendix via Monte Carlo simulations. In the third panel of tables 1 through 4 , we report on the empirical rejection frequencies and size of this periodic $\mathbf{J J}$ test method. From the results in these tables it is clear that the empirical size is very close to the nominal size, and hence we will use the fractiles in the appendix for the next empirical analysis.

The application of this periodic JJ procedure to the German consumption and income data, where we do not include additional lags in the first computation step, yields the following results:

$$
\begin{array}{llll}
\hat{\lambda}_{11}=0.423 & \hat{\lambda}_{21}=0.224 & \operatorname{Tr}_{1}(r \leq 1)=6.861 & \operatorname{Tr}_{1}(r \leq 0)=21.709^{* *} \\
\hat{\lambda}_{12}=0.270 & \hat{\lambda}_{22}=0.145 & \operatorname{Tr}_{2}(r \leq 1)=4.217 & \operatorname{Tr}_{2}(r \leq 0)=12.703 \\
\hat{\lambda}_{13}=0.166 & \hat{\lambda}_{23}=0.104 & \operatorname{Tr}_{3}(r \leq 1)=2.956 & \operatorname{Tr}_{3}(r \leq 0)=7.851 \\
\hat{\lambda}_{14}=0.199 & \hat{\lambda}_{24}=0.053 & \operatorname{Tr}_{4}(r \leq 1)=1.462 & \operatorname{Tr}_{4}(r \leq 0)=7.453
\end{array}
$$

where ${ }^{* *}$ indicates significant at a $5 \%$ level. These outcomes suggest that there seems to be cointegration between $c_{t}$ and $y_{t}$ in the first quarter only. This co- 
integration relation is estimated to be $C_{1 T}-0.978 Y_{1 T}$. A test for the hypothesis that this relaton equals $C_{1 T}-Y_{1 T}$ yields a value of 0.965 , which is insignificant when compared to the fractiles of the $\chi^{2}(1)$ distribution. See Johansen and Juselius (1990) for details of this test on restrictions on cointegration vectors.

The estimation of periodic VAR models of various orders and the application of several model selection criteria yields that a PVAR(1) is an empirically adequate model for $\left(c_{t}, y_{t}\right)$. This implies that the presence of a cointegration relationship in the first quarter means error correction in the second quarter, i.e. the model for the bivariate process $\left(c_{t}, y_{t}\right)$ is

$$
\begin{aligned}
& \left(1-\phi_{s} B\right) c_{t}=\mu_{s}+\pi_{1} D_{2 t}\left(c_{t-1}-y_{t-1}\right)+\varepsilon_{t} \\
& \left(1-\alpha_{s} B\right) y_{t}=\delta_{s}+\pi_{2} D_{2 t}\left(c_{t-1}-y_{t-1}\right)+v_{t}
\end{aligned}
$$

The parameters in this system can be estimated using nonlinear least squares since we impose the restrictions $\phi_{1} \phi_{2} \phi_{3} \phi_{4}=1$ and $\alpha_{1} \alpha_{2} \alpha_{3} \alpha_{4}=1$. The estimates for these $\phi_{s}$ and $\alpha_{s}$ are very much similar to those reported in section 2. More interesting are the results for the error correction parameters $\hat{\pi}_{1}=-0.373$ with a $t$ ratio of -3.148 , and $\hat{\pi}_{2}=-0.189$ with a $t$ ratio of -1.321 . Hence, there is only significant error correction in the model for consumption.

To summarize, we find empirical evidence of cointegration between German consumption and income in the first quarter only. This evidence is obtained using a two-step method which considers the stochastic trends constructed via the Box-Tiao method andusing the Johansen method when applied to a periodic VAR process.

\section{Power of the Various Cointegration Methods}

A final investigation we carry out in this paper concerns the power of the various cointegration testing methods proposed in this section. Of course, we can generate a variety of DGPs in order to check this power. However, given the empirical application considered in this paper, we limit ourselves to a DGP as (27) and (28), i.e. a PVAR(1) process with cointegration in the first quarter. Note that, given two PIAR(1) processes, cointegration in one quarter automatically implies cointegration in the other quarters. In practice, however, it may depend on the power of the test procedure whether one finds more than one cointegration relationship.

In table 6 we report on the empirical power of the various cointegration testing methods. Note that "power" here refers to the finding of a single cointegration relationship, i.e. for the $\mathbf{J} \mathbf{J}$ method we also exclude the occasions where more than one cointegration relationship is found. The results in table 6 clearly indicate that for this particular DGP the power of the "JJ after BT" method and the periodic JJ approach is highest, while that of the EG methods 
is typically smallest. A thourough comparison of the various methods using a wide range of DGPs will indicate whether the results in table 6 reflect a more general result. However, we consider such an extensive investigation to be outside the scope of the present paper.

\section{Concluding Remarks}

This paper deals with two empirical issues concerning econometric models for German consumption and income in the period 1960.1-1987.4. The first is an investigation into the seasonality and stochastic trend properties of the univariate series. The second is the proposal of methods to test cointegration between consumption and income, given that we find that both series can be described using first order periodic autoregressions with a single unit root. Given that such models allow a dependence of the seasonal pattern on the stochastic trend, we have to take account of this aspect when considering the series in a cointegration analysis. We propose several cointegration testing approaches and evaluate their empirical performance in Monte Carlo simulations. One of the approaches is an extension of the Johansen method to periodic VAR models. In this paper we analyse the application of the various methods to bivariate time series only. In principle, it seems that extensions to higher dimensional time series are straightforward. Finally, the empirical evidence obtained for the German time series suggests that there is cointegration between consumption and income in the first quarter only. Possible causes for this empirical result are that wage contracts in Germany negotiated for one year take effect at the beginning of each year. Furthermore, prices also typically change at the beginning of the year. This may establish error correction behavior of economic agents. However, further research into the origin of this specific periodic behavior seems needed.

Although this paper focuses on an empirical analysis of economic time series using several econometric techniques, there may also be implications for economic theory in case one finds periodic VAR models with periodically varying error correction mechanisms to be adequate data descriptions. For example, one may wish to extend economic theories to allow for periodically varying structures. An important study in this context is Osborn (1988), where parameters in a utility function for consumption can take time-varying values. More general, one may take into account that economic agents may incorporate changing seasonal patterns into their plans and expectations. If so, such behaviour implies that it may be more appropriate not to remove seasonal patterns via certain seasonal adjustment methods, but to model seasonality explicitly. The econometric methods analysed in the present paper may then be useful tools. 


\section{Appendix}

This appendix contains the critical values of the Johansen cointegration tests for a sample size of 25 observations. The quantiles are based on 10000 replications. The test statistics are computed from the original formulas in Johansen and Juselius (1990). The table corresponds to table A.2 in Johansen and Juselius (1990). The reader is referred to that paper for more details.

Table A1. Quantiles of the Johansen cointegration test statistics Sample size is 25. The data generating process contains no trend, and the constant term $\mu$ is unrestricted

\begin{tabular}{lrrrrrrrr}
\hline $\operatorname{dim}$ & $50 \%$ & $80 \%$ & $90 \%$ & $95 \%$ & $97.5 \%$ & $99 \%$ & mean & \multicolumn{1}{c}{ var } \\
\hline \multicolumn{2}{l}{ Maximal eigenvalue } & & & & & & & \\
1 & 2.43 & 4.93 & 6.70 & 8.29 & 9.91 & 12.09 & 3.06 & 7.36 \\
2 & 7.86 & 11.38 & 13.70 & 15.75 & 17.88 & 20.51 & 8.54 & 14.76 \\
Trace & & & & & & & & \\
1 & 2.43 & 4.93 & 6.70 & 8.29 & 9.91 & 12.09 & 3.06 & 7.36 \\
2 & 9.78 & 13.99 & 16.56 & 18.90 & 21.26 & 23.70 & 10.45 & 20.64 \\
\hline
\end{tabular}

Acknowledgements The first author thanks the Royal Netherlands Academy of Arts and Sciences and the second author thanks the Netherlands Organisation for Scientific Research (NWO) for financial support. The comments from participants at a seminar at Humboldt University Berlin, from Peter Boswijk, Helmut Herwartz, Søren Johansen and from two anonymous referees are gratefully acknowledged.

\section{References}

Boswijk HP, Franses PH (1994) Unit roots in periodic autoregressions. Tinbergen Institute Discussion Paper 94-4, Erasmus University Rotterdam

Box GPE, Tiao GC (1977) A canonical analysis of multiple time series. Biometrika 64:355-365

Engle RF, Granger CWJ (1987) Cointegration and error correction: Representation, estimation, and testing. Econometrica 55:251-76

Franses PH, Paap R (1993) Model selection in periodic autoregressions. Econometric Institute Report 9313, Oxford Bulletin of Economics and Statistics, to appear

Gonzalo J, Granger CWJ (1994) Estimation of common long-memory components in cointegrated systems. Journal of Business and Economic Statistics to appear

Hylleberg S, Engle RF, Granger CWJ, Yoo BS (1990) Seasonal integration and cointegration. Journal of Econometrics 44:215-238

Johansen S (1991) Estimation and hypothesis testing of cointegration vectors in Gaussian vector autoregressive models. Econometrica 59:1551-80

Johansen S, Juselius K (1990) Maximum likelihood estimation and inference on cointegration with applications to the demand for money. Oxford Bulletin of Economics and Statistics $52: 169-210$ 
Jones RH, Brelsford WM (1967) Time series with periodic structure. Biometrika 54:403-408 Lütkepohl H (1991) Introduction to multiple time series analysis, Berlin Springer Verlag MacKinnon JG (1991) Critical values for cointegration tests. In: Engle RF, Granger CWJ (eds) Long-run economic relationships: Readings in cointegration, Oxford University Press

Osborn DR (1988) Seasonality and habit persistence in a Life-Cycle model of consumption. Journal of Applied Econometrics 3:255-266

Pagano M (1978) On periodic and multiple autoregressions. Annals of Statistics 6:1310-1317

First version received: August 1993

Final version received: November 1994 